This item was submitted to Loughborough's Research Repository by the author.

Items in Figshare are protected by copyright, with all rights reserved, unless otherwise indicated.

\title{
Event-triggered output consensus disturbance rejection algorithm for multi- agent systems with time-varying disturbances
}

PLEASE CITE THE PUBLISHED VERSION

https://doi.org/10.1016/j.jfranklin.2020.04.014

PUBLISHER

Elsevier

VERSION

AM (Accepted Manuscript)

\section{PUBLISHER STATEMENT}

This paper was accepted for publication in the journal Journal of the Franklin Institute and the definitive published version is available at https://doi.org/10.1016/j.jfranklin.2020.04.014.

LICENCE

CC BY-NC-ND 4.0

\section{REPOSITORY RECORD}

Sun, Jiankun, Jun Yang, Shihua Li, Xiangyu Wang, and Guipu Li. 2020. "Event-triggered Output Consensus Disturbance Rejection Algorithm for Multi-agent Systems with Time-varying Disturbances”. Loughborough University. https://hdl.handle.net/2134/16818925.v1. 


\title{
Event-Triggered Output Consensus Disturbance Rejection Algorithm for Multi-Agent Systems With Time-Varying Disturbances
}

\author{
Jiankun Sun ${ }^{\mathrm{a}}$, Jun Yang ${ }^{\mathrm{b}, *}$, Shihua $\mathrm{Li}^{\mathrm{b}}$, Xiangyu Wang ${ }^{\mathrm{b}}$, Guipu $\mathrm{Li}^{\mathrm{b}}$ \\ ${ }^{a}$ School of Artificial Intelligence and Automation, Huazhong University of Science and Technology, Key Laboratory of Image Processing and In- \\ telligent Control of Education Ministry of China, Wuhan 430074, P. R. \\ China \\ ${ }^{b}$ School of Automation, Southeast University, Key Laboratory of Measurement and Control of CSE, Ministry of Education, Nanjing 210096, P. $R$. \\ China
}

\begin{abstract}
This study investigates the problem of event-triggered output consensus disturbance rejection for multi-agent systems subject to time-varying disturbances. By virtue of the reduced-order generalized proportional-integral observer (GPIO) technique, a new output consensus disturbance rejection protocol is developed based on the measurement outputs. Taking the limited communication bandwidth into account, the multi-agent system closes the loop under the proposed consensus protocol only when a distributed event-triggering mechanism decides to transmit agent's current output to its neighbors. The proposed output consensus protocol can effectively enhance the robustness against the time-varying disturbances and save the communication resource, since the time-varying disturbances are accurately estimated and compensated. Furthermore, the proposed consensus algorithm does not require continuous communication among the neighboring agents and can successfully avoid the Zeno phenomenon. Finally, the numerical simulation results are presented to verify the effectiveness of the proposed output consensus protocol.
\end{abstract}

Keywords: Event-triggered output consensus disturbance rejection, time-varying disturbances, reduced-order GPIO, multi-agent systems.

\section{Introduction}

The consensus problem of multi-agent systems has attracted lots of attention in recent several decades [1-3]. The consensus problem is solved under some algorithms if an agreement on the states of interest is eventually achieved for all the agents. Multi-agent systems generally consist of several distributed components including sensors, controllers and actuator nodes, which are connected over a shared communication network [4-6]. In addition, each agent and its neighbors exchange the information with each other if there exists an accessible communication channel, in order to accomplish some complex tasks among several distributed processors [7].

It is well known that a pre-specified sampling rate is set for the traditional time-triggered control strategy [8]. Even though such a control strategy is easily designed and the theoretical analysis is quite mature, it can be observed that the time-triggered control could give rise to a wasteful use of communication resource since the control input is periodically updated without considering the performance requirement. Such a situation forces researchers to consider how to design the resource-aware control method while taking the costly communication resource into account [9].

A promising solution to save the communication resource is to execute the control tasks according to the performance requirement instead of the elapse of a fixed sampling rate [10-12]. Based on the principle, a novel control strategy called event-triggered control has been developed such as to reduce the conflict between the limited communication resource and the transmission times [13-16]. The main merit of the event-triggered control is that it provides

\footnotetext{
${ }^{*}$ Corresponding author

Email addresses: jksun@hust. edu.com (Jiankun Sun), j.yang84@ seu. edu. cn (Jun Yang), Ish@ seu.edu.cn (Shihua Li), w.x.y@seu.edu.cn (Xiangyu Wang), liguipucd@126.com (Guipu Li)
} 
one freedom to regulate the communication transmission rate in the controller design. Some results on event-triggered consensus protocol have been shown in [17-20] and the references therein. Most of the above-mentioned results are limited to the hypothesis that the consensus protocols can obtains the full states of agents. In practice, it may be more realistic when only partial states can be available [1,21]. For example, in order to save the costs, most of commercially available robot manipulators do not equip the velocity sensors, thus the velocity measurement is generally unavailable [22], or the velocity can not be precisely measured even the sensor is equipped. To reconstruct the system states, the reduced-order observer has been proposed and becoming more and more attractive, since less additional dynamics and less parameters needs to be regulated for reduced-order observer, hence it is easier to implement. Despite, there is few result on the reduced-order observer based consensus problem, except for [23].

Moreover, the accurate system dynamics can not be obtained because various disturbances are inevitable in practical applications [24-30]. To tackle the issue, many consensus output regulation methods and consensus disturbance rejection algorithms have been developed for multi-agent systems in the presence of disturbances [31-35]. Disturbances of the event-triggered control systems not only have an undesirable influence on the control performance, but also on the communication property. In $[15,36]$, it has been shown that disturbances may leads to more transmission times. What is worse, the Zeno behavior may be induced by an arbitrarily small external disturbance. To address the issue, $[14,15]$ proposed the event-triggered disturbance rejection control strategies by integrating the disturbance estimation and compensation technique in the design of controller for single networked control systems, such that both the communication property and the control performance can be significantly improved. However, for multiagent systems with time-varying disturbances, it still open for the problem of the reduced-order observer based output consensus disturbance rejection via event-triggering mechanism.

This paper studies the event-triggered output consensus disturbance rejection problem of second-order multi-agent systems with time-varying disturbances. When only the output is available, a reduced-order generalized proportionalintegral observer (GPIO) is first employed to reconstruct the unknown state and the time-varying disturbance for each agent, which does not need to know the accurate disturbance model. Then, we propose a new event-triggered output consensus disturbance rejection algorithm by using the estimates. To save the limited communication resource, a distributed event-triggering scheme is proposed to determines whether or not to transmit the newest measurement output to its neighbors. The analysis is presented to guarantee the avoidance of that the Zeno behavior. The main novelties of the proposed output consensus protocol are described as follows:

1) By virtue of disturbance estimation and compensation technique, the proposed event-triggered output consensus disturbance rejection algorithm can effectively reject the undesirable influence of time-varying disturbances on both of the consensus performance and the communication property.

2) Compared with some existing works $[18,37]$ on event-triggered consensus algorithms, where the continuous communication among each agent and its neighbors is necessary, the proposed event-triggered output consensus disturbance rejection algorithm only requires the sampled information from the neighboring agents and is easier to be implemented in digital platform.

3) In contrast with the traditional full-order observer [14, 26], the proposed output consensus protocol is designed via distributed reduced-order observers, which do not need to estimate the known states, and can significantly enhance the estimation performance by increasing the bandwidth of observer in the absence of measurement noise [38].

In the rest of this paper, Section II gives the formulation description and the problem statement, and Section III presents the consensus protocol design and the main results. Then, the simulation results are exhibited to verify the effectiveness of the proposed consensus protocol in Section IV. In final, Section V gives the main conclusions.

Notation: Throughout this study, some standard notations are defined as follows: The matrix transpose is represented by the superscript $T . \mathbb{R}$ and $\mathbb{N}$ denote the sets of real numbers and nonnegative integers, respectively. $|r|$ stands for the absolute value of a scalar $r \in \mathbb{R},\|\cdot\|$ denotes the Euclidean norm of a vector and the corresponding induced matrix norm. Given two matrices $A$ and $B$, the Kronecker product of the two matrices is defined by $A \otimes B$. The all-one column vector is denoted by $\mathbf{1}_{N}$, and the identity matrix is represented by $I_{N}$ with dimension $N$. 


\section{Preliminaries}

\subsection{Graph Theory Notions}

Given an undirected graph $G=(\mathscr{V}, \mathscr{E}, \mathscr{A})$, where $\mathscr{V}=\left\{a_{i}, i=1, \cdots, N\right\}$ represents the set of nodes, $\mathscr{E} \subseteq \mathscr{V} \times \mathscr{V}$ denotes the set of edges, and $\mathscr{A}$ is the weighted adjacency matrix of the graph $G$. The node indexes belong to a finite index set $\Gamma=\{1, \cdots, N\}$. An edge $\left(a_{i}, a_{j}\right)$ denotes that nodes $a_{i}$ and $a_{j}$ can exchange information with each other via an available communication channel. In addition, a path for the undirected graph $G$ is a sequence of $a_{k_{1}}, a_{k_{2}}, \cdots, a_{k_{m}}$ with finite nodes from $a_{k_{1}}$ to $a_{k_{m}}$, such that $\left(a_{k_{l}}, a_{k_{l+1}}\right) \in \mathscr{E}$ for $l=1,2, \cdots, m-1$. The adjacency matrix $\mathscr{A}=\left[a_{i j}\right] \in$ $\mathbb{R}^{N \times N}$ associated with the graph $G$ is defined such that $a_{i j}=a_{j i}>0$ if $\left(a_{i}, a_{j}\right) \in \mathscr{E}$ while $a_{i j}=a_{j i}=0$ otherwise. It is assumed that $a_{i i}=0, \forall i \in \Gamma$. The Laplacian matrix $L=\left[l_{i j}\right] \in \mathbb{R}^{N \times N}$ associated with $\mathscr{A}$ is defined as $l_{i i}=\sum_{j=1}^{N} a_{i j}$ and $l_{i j}=-a_{i j}$, where $i \neq j$. Note that matrix $L$ is symmetric for the undirected graph $G$. An undirected graph is connected if there is an undirected path between every pair of distinct nodes.

\subsection{Problem Formulation}

Given a class of second-order multi-agents systems with time-varying disturbances as follows:

$$
\left\{\begin{array}{l}
\dot{x}_{i}(t)=v_{i}(t), \\
\dot{v}_{i}(t)=u_{i}(t)+d_{i}(t), \\
y_{i}(t)=x_{i}(t), i \in \Gamma,
\end{array}\right.
$$

where $x_{i} \in \mathbb{R}, v_{i} \in \mathbb{R}, y_{i}$ and $u_{i} \in \mathbb{R}$ are the position state, the velocity state, the measurement output and the consensus protocol of agent $i$, respectively. $d_{i} \in \mathbb{R}$ is the time-varying disturbance in agent $i . N$ is the number of agents. The multi-agent systems are connected with an undirected communication graph $G$.

The aim of this study is to design a new event-triggered output consensus disturbance rejection protocol, such that the practical output consensus can be achieved by all the agents in (1), and the undesirable influence of time-varying disturbances can be effectively rejected for both the communication property and the consensus performance. Then, we give a definition of the practical output consensus as follows:

Definition 1 If for any initial condition and any given $\alpha>0$, it has

$$
\lim _{t \rightarrow \infty}\left|y_{i}(t)-y_{j}(t)\right| \leq \alpha, \forall i, j=1, \cdots, N
$$

then the multi-agent system (1) achieves the practical output consensus under the consensus protocol $u_{i}$.

Then, we introduce some assumptions and useful lemmas, which play a key role in the consensus analysis part.

Assumption 1 For the time-varying disturbance $d_{i}(t)$ of $\left.i \in \Gamma, 1\right)$ at least the first $(q-1)$-th derivatives of $d_{i}(t)$ exist, where $q \geq 2$; 2) let $d_{i}^{(j)}(t)$ stands for the $j$-th derivative of $d_{i}(t) . \forall i \in \Gamma$, the $j$-th derivative of $d_{i}(t)$ is uniformly bounded, i.e., $\left|d_{i}^{(j)}(t)\right| \leq \bar{d}, \forall t \geq 0$ and $j=1, \cdots, q-1$, where $\bar{d}$ is a positive constant.

Assumption 2 The undirected communication graph $G$ is connected.

Lemma 1 [39] If an undirected graph $G$ is connected, then its Laplacian matrix L has a simple eigenvalue 0 with the associated eigenvector $\mathbf{1}_{N}$ and all the other eigenvalues are positive. Let $\lambda_{1}, \lambda_{2}, \cdots, \lambda_{N}$ be the eigenvalues of the Laplacian matrix $L$, then one has $0=\lambda_{1}<\lambda_{2} \leq \cdots \leq \lambda_{N}$, which means that $L$ is positive semi-definite. For any $x=\left[x_{1}, \cdots, x_{N}\right]^{T} \in \mathbb{R}^{N}$, one has $x^{T} L x=\frac{1}{2} \sum_{i=1}^{N} \sum_{j=1}^{N} a_{i j}\left(x_{i}(t)-x_{j}(t)\right)^{2}$. Furthermore, if $\mathbf{1}_{N}^{T} x=0$, then $x^{T} L x \geq \lambda_{2} x^{T} x$.

Lemma 2 [40] Given any matrices A,B,C and D with appropriate dimensions, the Kronecker product $\otimes$ has the following properties:

1) $(A+B) \otimes C=A \otimes C+A \otimes B$,

2) $(A \otimes B)(C \otimes D)=(A C) \otimes(C D)$, 


\section{3) $(A \otimes B)^{T}=A^{T} \otimes B^{T}$.}

According to the idea of disturbance estimation and compensation technique [14, 26], the time-varying disturbance $d_{i}(t)$ can be viewed as a lumped disturbance, which includes exogenous disturbance, unmodeled dynamics, nonlinear uncertainties, etc. Hence, it is almost impossible to measure the lumped disturbance $d_{i}(t)$ by a sensor, which motivates us to design the distributed reduced-order GPIOs to estimate the unknown disturbances.

Remark 1 It should be mentioned that Assumption 1 is very common in the domain of disturbance estimation and compensation based control [25]. Actually, the disturbance under Assumption 1 covers unknown constant and ramp signal as special cases. The underlying idea is that we can use the finite terms of Taylor series to locally approximate the time-varying disturbance [41]. For the simplicity's sake, the time-varying disturbances $d_{i}(t)$ of different agents are assumed to satisfy the same condition given in Assumption 1. However, we can easily extend the results in this paper to the case where the minimum differentiable order $(q-1)$ of $d_{i}(t)$ or the bound $\theta$ is not the same for different agents.

\section{Main Results}

\subsection{Event-Triggered Output Consensus Disturbance Rejection Protocol}

Assume that only the measurement output $y_{i}$ is known for each agent $i$. In order to reconstruct the time-varying disturbances and the unknown states, a distributed reduced-order GPIO for agent $i$ is designed by

$$
\left\{\begin{array}{l}
\dot{z}_{i, 1}(t)=-\beta_{1}\left(z_{i, 1}(t)+\beta_{1} y_{i}(t)\right)+z_{i, 2}(t)+\beta_{2} y_{i}(t)+u_{i}(t), \\
\quad \vdots \\
\dot{z}_{i, 2}(t)=-\beta_{2}\left(z_{i, 1}(t)+\beta_{1} y_{i}(t)\right)+z_{i, 3}(t)+\beta_{3} y_{i}(t), \\
\dot{z}_{i, q-1}(t)=-\beta_{q-1}\left(z_{i, 1}(t)+\beta_{1} y_{i}(t)\right)+z_{i, q}(t)+\beta_{q} y_{i}(t), \\
\dot{z}_{i, q}(t)=-\beta_{q}\left(z_{i, 1}(t)+\beta_{1} y_{i}(t)\right), \\
\hat{v}_{i}(t)=z_{i, 1}(t)+\beta_{1} x_{i}(t), \hat{d}_{i}(t)=z_{i, 2}(t)+\beta_{2} x_{i}(t), \cdots, \\
\hat{d}_{i}^{(q-2)}(t)=z_{i, q}(t)+\beta_{q} x_{i}(t),
\end{array}\right.
$$

where $z_{i, j}, j=1, \cdots, q$ are the internal variables of (2) for agent $i$. $\hat{v}_{i}$ and $\hat{d}_{i}^{(j)}, j=0, \cdots, q-2$ denote the estimates of the state $v_{i}$ and the first $j$-th derivatives of $d_{i}$, respectively. $\beta_{i}, i=1, \cdots, q$ are the coefficients such that the polynomial $f(s)=s^{q}+\beta_{1} s^{q-1}+\cdots+\beta_{q}$ is Hurwitz.

Remark 2 It should be mentioned that under the classic full-order observer (see $[14,26])$, there will be $(q+1)$ dynamics to be designed and $(q+1)$ parameters to be regulated, i.e., the position, the velocity, and the first $(q-1)$ th derivatives of disturbance need to be estimated. However, for the proposed reduced-order observer (2), only the velocity and the first $(q-1)$ th derivatives of $d_{i}(t)$ need to be estimated since the position is available, and there are only $q$ parameters to be regulated and $q$ dynamics to be designed. Moreover, in contrast with the full-order observer, the proposed reduced-order observer has better transient response under the same bandwidth [38] in the absence of measurement noise.

Let $e_{i}=\left[e_{i, 1}, \cdots, e_{i, q}\right]^{T}$ with $e_{i, 1}=v_{i}-\hat{v}_{i}, e_{i, 2}=d_{i}-\hat{d}_{i}, \cdots, e_{i, q}=d_{i}^{(q-2)}-\hat{d}_{i}^{(q-2)}$. The dynamics of the estimation error are obtained as follows:

$$
\dot{e}_{i}(t)=A_{e} e_{i}(t)+B_{d} d_{i}^{(q-1)}(t), i \in \Gamma,
$$

where $A_{e}=\left[\begin{array}{ccccc}-\beta_{1} & 1 & 0 & \cdots & 0 \\ -\beta_{2} & 0 & 1 & \cdots & 0 \\ \vdots & \vdots & \vdots & \ddots & \vdots \\ -\beta_{q-1} & 0 & 0 & \cdots & 1 \\ -\beta_{q} & 0 & 0 & \cdots & 0\end{array}\right] \in \mathbb{R}^{q \times q}, B_{d}=[0, \cdots, 0,1]^{T} \in \mathbb{R}^{q \times 1}$. 


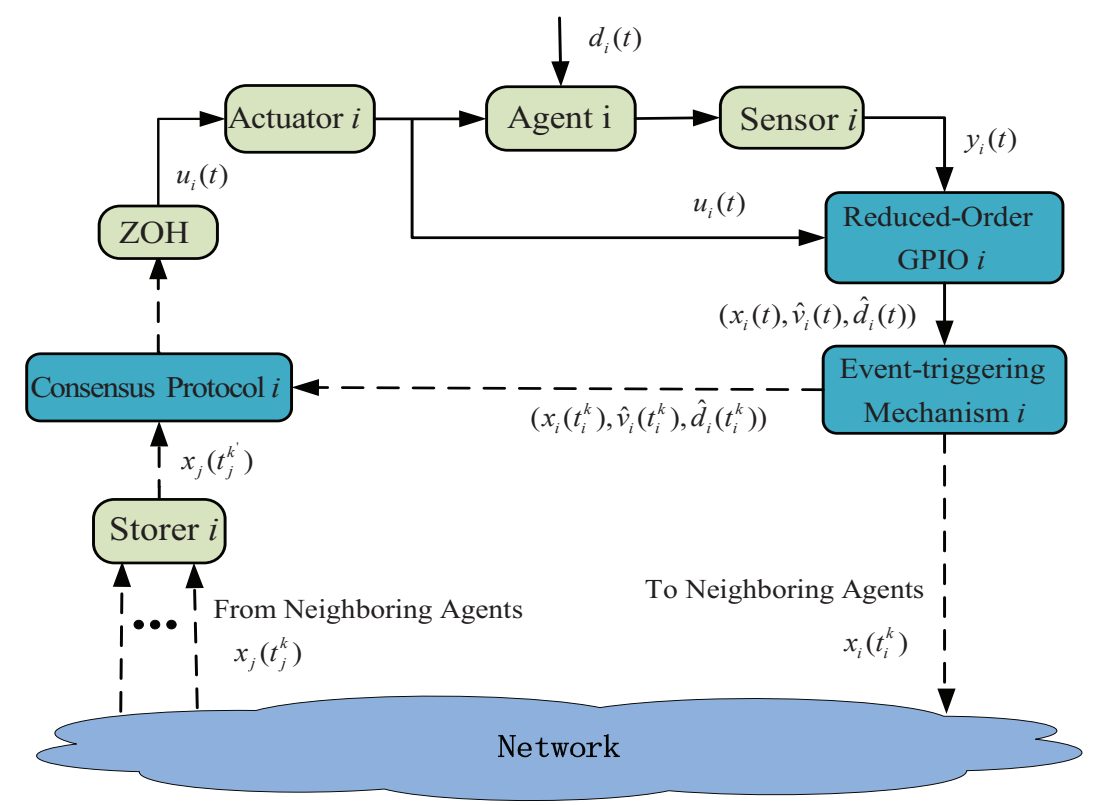

Figure 1: The framework of the proposed consensus algorithm based on reduced-order GPIO, where the signals are transmitted continuously along the solid lines, intermittently based on the events along the dashed line.

For agent $i,\left\{t_{i}^{k}\right\}_{k \in \mathbb{N}}$ represents the set of the event-triggering instants, where $t_{i}^{0}=0$ and $t_{i}^{k}<t_{i}^{k+1}$. With the help of some new error variables $\xi_{i}^{x}(t)=x_{i}\left(t_{i}^{k}\right)-x_{i}(t), \xi_{i}^{v}(t)=\hat{v}_{i}\left(t_{i}^{k}\right)-\hat{v}_{i}(t)$ and $\xi_{i}^{d}(t)=\hat{d}_{i}\left(t_{i}^{k}\right)-\hat{d}_{i}(t), t_{i}^{k} \leq t<t_{i}^{k+1}$, a new distributed event-triggering mechanism is designed for agent $i$ by

$$
t_{i}^{k+1}=\inf \left\{t \geq t_{i}^{k} \mid \xi_{i}^{T}(t) \Phi_{i} \xi_{i}(t) \geq \mu_{0} e^{-\mu_{1} t}+\mu_{2}\right\},
$$

where $\xi_{i}(t)=\left[\xi_{i}^{x}(t), \xi_{i}^{v}(t), \xi_{i}^{d}(t)\right]^{T}$. The weighting matrix $\Phi_{i}$ is positive definition. The absolute threshold parameters $\mu_{0}, \mu_{1}$ and $\mu_{2}$ are three positive constants to be regulated. In the proposed event-triggering mechanism (4), the disturbance estimation error $\xi_{i}^{d}(t)=\hat{d}_{i}\left(t_{i}^{k}\right)-\hat{d}_{i}(t)$ is used since it has a serious influence on the consensus performance.

By using the measurement outputs and the estimates at the event-triggering instants, an event-triggered output consensus disturbance rejection protocol for agent $i$ is developed as follows:

$$
u_{i}(t)=-k_{1} k_{2} \sum_{j=1}^{N} a_{i j}\left(x_{i}\left(t_{i}^{k}\right)-x_{j}\left(t_{j}^{k_{j}^{\prime}(t)}\right)\right)-k_{1} \hat{v}_{i}\left(t_{i}^{k}\right)-\hat{d}_{i}\left(t_{i}^{k}\right), t \in\left[t_{i}^{k}, t_{i}^{k+1}\right),
$$

where the control parameters $k_{1}>0$ and $k_{2}>0 . k_{j}^{\prime}(t) \triangleq \arg \min _{m}\left\{t-t_{j}^{m} \mid t \geq t_{j}^{m}, m \in \mathbb{N}\right\}, j \in \Gamma$.

In Fig. 1, it describes the framework of the proposed output consensus disturbance rejection algorithm. The unknown velocities and the time-varying disturbances are first estimated by the proposed reduced-order GPIOs (2). Then, the proposed event-triggering mechanism (4) decides whether the agent should transmit its current information to its neighboring agents via the shared communication network or not. Once the latest sampled information is received by the computer, the control signal will be updated according to (5).

Remark 3 For the sake of simplicity, the parameters $\mu_{0}, \mu_{1}$ and $\mu_{2}$ in (4) are chosen to be the same for each eventtriggering condition in this paper. However, we can easily extend the results in this paper to the case where the parameters are different for each agent. The weighting matrix $\Phi_{i}$ can be prudently chosen to regulate the weight of the different entry of the error vector $\xi_{i}(t)$. In the simulation and experiment, it is suggested that the weighting matrix $\Phi_{i}$ should be regulated in a manner of trial and error to obtain a better balance between the communication property and the consensus performance. 
Remark 4 In the proposed event-triggered consensus protocol (5), it can be observed that each agent only requires to know the sampled measurement output from its neighbors at the event-triggering instants. Hence, the continuous communication among neighbors is not required. Such a design method is much more efficient in digital communication compared with the existing results $[18,37]$, where the communication among neighbors is essentially continuous.

\subsection{Consensus Analysis}

It is obvious that the observer gains $\beta_{i}, i=1, \cdots, q$ can be chosen such that $A_{e}$ is Hurwitz. Then, given a positive constant $\lambda, A_{e}^{T} Q+Q A_{e}=-\lambda I_{q}$ has a unique solution $Q=Q^{T} \in \mathbb{R}^{q \times q}$ that is a positive definite matrix.

Theorem 1 When the multi-agent systems (1) satisfy Assumptions 1 and 2, under the proposed output consensus protocol (2), (5), and (4), if the parameters are chosen such that

$$
\begin{aligned}
& k_{2} \geq \frac{1}{2}+\frac{\beta+\gamma N}{2}+\sigma \\
& k_{1} \geq k_{2}^{2}\left(\frac{1+\gamma N}{2}+\frac{3 \beta+2 \gamma N}{2 k_{2}}+\frac{3}{2 k_{2}^{2}}+\sigma\right), \\
& \lambda \geq 2\left(\frac{1}{2}+\frac{k_{1}^{2}}{2 k_{2}^{2}}+\frac{1}{2 k_{2}^{2}}+\sigma\right),
\end{aligned}
$$

where $\sigma$ is a positive constant, $\beta=\max _{i \in \Gamma}\left\{\sum_{j=1}^{N} a_{i j}\right\}$ and $\gamma=\max _{i, j \in \Gamma}\left\{a_{i j}\right\}$, then the estimation errors and the output consensus errors asymptotically converge to a ball with radius $\frac{\left.\sigma_{2}\left(2 \sigma_{1} \tilde{\mu}+N \| Q B_{d}\right) \|^{2} \vec{d}^{2}\right)}{2 \sigma}$ around the origin, where $\tilde{\mu}=\sum_{i=1}^{N} \frac{\mu_{2}}{\lambda_{\min }\left(\Phi_{i}\right)}, \sigma_{1}=\max \left\{\frac{\bar{l} N k_{1}^{2}}{2}, \frac{k_{1}^{2}}{2 k_{2}^{2}}, \frac{1}{2 k_{2}^{2}}\right\}, \sigma_{2}=\max \left\{\frac{1}{2 \lambda_{2}}, \frac{1}{2 k_{2}^{2}}, \frac{1}{2 \lambda_{M}(Q)}\right\}$ and $\bar{l}=\max _{i, j \in \Gamma}\left\{l_{i j}^{2}\right\}$. Moreover, by selecting appropriate parameters, the bound can be adjusted to be arbitrarily small, which means that the practical output consensus problem is solved under the proposed consensus protocol.

Proof. The proof combines three steps. First, we design a virtual velocity for each agent, such that the practical output consensus can be asymptotically achieved. Second, the event-triggered controller (5) is designed to realize the asymptotic practical stabilization of the tracking error, which is the difference between the virtual velocity and the real velocity. Finally, combining with the error systems (3), a delicate analysis is given to demonstrate that the estimation errors and the output consensus errors asymptotically tend to a bounded region around the origin .

Step 1: With the help of definitions $x=\left[x_{1}, \cdots, x_{N}\right]^{T}$ and defining a new variable $p=L x=\left[p_{1}, \cdots, p_{N}\right]^{T}$, we have that $p_{i}=\sum_{j=1}^{N} a_{i j}\left(x_{i}-x_{j}\right), i \in \Gamma$. Considering the multi-agent systems (1), we construct a candidate Lyapunov function by

$$
V_{0}(t)=\frac{1}{2} x^{T}(t) L x(t)=\frac{1}{4} \sum_{i=1}^{N} \sum_{j=1}^{N} a_{i j} \eta_{i j}^{2}(t) \geq 0,
$$

where $\eta_{i j}=x_{i}-x_{j}, i, j \in \Gamma$.

We take the derivative of $V_{0}(t)$ along (1) and get

$$
\dot{V}_{0}(t)=x^{T}(t) L \dot{x}(t)=\sum_{i=1}^{N} \sum_{j=1}^{N} a_{i j} \eta_{i j}(t) v_{i}(t)=\sum_{i=1}^{N} p_{i}(t) v_{i}(t) .
$$

Defining $v_{i}^{*}=-k_{2} p_{i}$ as the virtual input for agent $i$, one obtains

$$
\begin{aligned}
\dot{V}_{0}(t) & =-k_{2} \sum_{i=1}^{N}\left|p_{i}(t)\right|^{2}+\sum_{i=1}^{N} p_{i}(t)\left(v_{i}(t)-v_{i}^{*}(t)\right) \\
& \leq-\left(k_{2}-\frac{1}{2}\right) \sum_{i=1}^{N}\left|p_{i}(t)\right|^{2}+\frac{1}{2} \sum_{i=1}^{N}\left|v_{i}(t)-v_{i}^{*}(t)\right|^{2}
\end{aligned}
$$


Step 2: Let $V_{x}=V_{0}+\sum_{i=1}^{N} V_{i}$ with $V_{i}=\frac{1}{2 k_{2}^{2}}\left(v_{i}-v_{i}^{*}\right)^{2}, i \in \Gamma$. Taking the time derivative of $V_{i}(t)$ along (1), it has

$$
\dot{V}_{i}(t)=-\frac{1}{k_{2}^{2}} \frac{\mathrm{d} v_{i}^{*}(t)}{\mathrm{d} t}\left(v_{i}(t)-v_{i}^{*}(t)\right)+\frac{1}{k_{2}^{2}}\left(v_{i}(t)-v_{i}^{*}(t)\right)\left(u_{i}(t)+d_{i}(t)\right), i \in \Gamma .
$$

It can be easily deduced that $\frac{\mathrm{d} v_{i}^{*}(t)}{\mathrm{d} t}=-k_{2} \sum_{j=1}^{N} a_{i j}\left(v_{i}(t)-v_{j}(t)\right) \leq k_{2}\left(\beta\left|v_{i}(t)\right|+\gamma \sum_{m=1}^{N}\left|v_{m}(t)\right|\right)$, where $\beta$ and $\gamma$ are defined in the statement of Theorem 1. It follows from (10) that

$$
\begin{aligned}
\dot{V}_{i}(t) \leq & \frac{1}{k_{2}} \beta\left|v_{i}(t)\right|\left|v_{i}(t)-v_{i}^{*}(t)\right|+\frac{1}{k_{2}} \gamma \sum_{m=1}^{N}\left|v_{m}(t)\right|\left|v_{i}(t)-v_{i}^{*}(t)\right| \\
& +\frac{1}{k_{2}^{2}}\left(v_{i}(t)-v_{i}^{*}(t)\right)\left(u_{i}(t)+d_{i}(t)\right) .
\end{aligned}
$$

With $v_{i}^{*}=-k_{2} p_{i}$ in mind, we obtain

$$
\begin{aligned}
\left|v_{m}(t)\right|\left|v_{i}(t)-v_{i}^{*}(t)\right| & \leq\left|v_{m}(t)-v_{m}^{*}(t)\right|\left|v_{i}(t)-v_{i}^{*}(t)\right|+\left|v_{m}^{*}(t)\right|\left|v_{i}(t)-v_{i}^{*}(t)\right| \\
& \leq \frac{\left(1+k_{2}\right)}{2}\left|v_{i}(t)-v_{i}^{*}(t)\right|^{2}+\frac{1}{2}\left|v_{m}(t)-v_{m}^{*}(t)\right|^{2}+\frac{k_{2}}{2}\left|p_{m}(t)\right|^{2} .
\end{aligned}
$$

Substituting (12) into (11) leads to

$$
\begin{aligned}
\dot{V}_{i}(t) \leq & \frac{\theta}{k_{2}}\left|v_{i}(t)-v_{i}^{*}(t)\right|^{2}+\frac{\gamma}{2 k_{2}} \sum_{m=1}^{N}\left|v_{m}(t)-v_{m}^{*}(t)\right|^{2} \\
& +\frac{1}{k_{2}^{2}}\left(v_{i}(t)-v_{i}^{*}(t)\right)\left(u_{i}(t)+d_{i}(t)\right)+\frac{\gamma}{2} \sum_{m=1}^{N}\left|p_{m}(t)\right|^{2}+\frac{\beta}{2}\left|p_{i}(t)\right|^{2}
\end{aligned}
$$

where $\theta=\frac{N \gamma\left(1+k_{2}\right)}{2}+\frac{3 \beta}{2}$.

In addition, the proposed output consensus protocol (5) can be furthermore depicted by $u_{i}=-k_{1}\left(v_{i}-v_{i}^{*}\right)-$ $k_{1} k_{2} \sum_{j=1}^{N} a_{i j}\left(\xi_{i}^{x}-\xi_{j}^{x}\right)-k_{1} \xi_{i}^{v}-\xi_{i}^{d}+k_{1} e_{i, 1}-\hat{d}_{i}, i \in \Gamma$. Then, the following inequality holds,

$$
\begin{aligned}
\dot{V}_{x}(t) \leq & -\left(k_{2}-\frac{1}{2}-\frac{\beta+\gamma N}{2}\right) \sum_{i=1}^{N}\left|p_{i}\right|^{2}-\left(\frac{k_{1}}{k_{2}^{2}}-\frac{1}{2}-\frac{\theta}{k_{2}}-\frac{\gamma N}{2 k_{2}}\right) \sum_{i=1}^{N}\left|v_{i}-v_{i}^{*}\right|^{2} \\
& -\frac{1}{k_{2}} \sum_{i=1}^{N}\left(v_{i}-v_{i}^{*}\right) k_{1} \sum_{j=1}^{N} l_{i j} \xi_{j}^{x}(t)-\frac{1}{k_{2}^{2}} \sum_{i=1}^{N}\left(v_{i}-v_{i}^{*}\right)\left(k_{1} \xi_{i}^{v}(t)+\xi_{i}^{d}(t)\right) \\
& +\frac{1}{k_{2}^{2}} \sum_{i=1}^{N}\left(v_{i}-v_{i}^{*}\right)\left(k_{1} e_{i, 1}(t)+e_{i, 2}(t)\right) .
\end{aligned}
$$

Step 3: Define $V_{e}=\frac{1}{2} e^{T}\left(I_{N} \otimes Q\right) e$ with $e=\left[e_{1}, \cdots, e_{N}\right]^{T}$. Taking the time derivative of $V_{e}(t)$ along (3) yields

$$
\begin{aligned}
\dot{V}_{e}(t)= & \frac{1}{2} e^{T}(t)\left(\left(I_{N} \otimes A_{e}\right)^{T}\left(I_{N} \otimes Q\right)+\left(I_{N} \otimes Q\right)\left(I_{N} \otimes A_{e}\right)\right) e(t) \\
& +e^{T}(t)\left(\left(I_{N} \otimes Q\right)\left(I_{N} \otimes B_{d}\right)\right) d^{(p-1)}(t)
\end{aligned}
$$

By Lemma 2, noting that $A_{e}^{T} Q+Q A_{e}=-\lambda I_{q}$, we further obtain

$$
\begin{aligned}
\dot{V}_{e}(t) & =-\frac{\lambda}{2} e^{T}(t) e(t)+e^{T}(t)\left(I_{N} \otimes Q B_{d}\right) d^{(q-1)}(t) \\
& \leq-\left(\frac{\lambda-1}{2}\right) e^{2}(t)+\frac{\left\|Q B_{d}\right\|^{2}}{2} \sum_{i=1}^{N}\left|d_{i}^{(q-1)}(t)\right|^{2} .
\end{aligned}
$$


Combining (14) and (16) together, we have

$$
\begin{aligned}
\dot{V}(t) \leq & -\left(k_{2}-\frac{1}{2}-\frac{\beta+\gamma N}{2}\right) \sum_{i=1}^{N}\left|p_{i}\right|^{2}-\left(\frac{k_{1}}{k_{2}^{2}}-\frac{1}{2}-\frac{\theta}{k_{2}}-\frac{\gamma N}{2 k_{2}}\right) \sum_{i=1}^{N}\left|v_{i}-v_{i}^{*}\right|^{2} \\
& -\left(\frac{\lambda-1}{2}\right) e^{2}(t)+\frac{\left\|Q B_{d}\right\|^{2}}{2} \sum_{i=1}^{N}\left|d_{i}^{(q-1)}(t)\right|^{2}-\frac{1}{k_{2}} \sum_{i=1}^{N}\left(v_{i}-v_{i}^{*}\right) k_{1} \sum_{j=1}^{N} l_{i j} \xi_{j}^{x}(t) \\
& -\frac{1}{k_{2}^{2}} \sum_{i=1}^{N}\left(v_{i}-v_{i}^{*}\right)\left(k_{1} \xi_{i}^{v}(t)+\xi_{i}^{d}(t)\right)+\frac{1}{k_{2}^{2}} \sum_{i=1}^{N}\left(v_{i}-v_{i}^{*}\right)\left(k_{1} e_{i, 1}(t)+e_{i, 2}(t)\right) .
\end{aligned}
$$

Recalling the definition of $\theta$, we have $\frac{1}{2}+\frac{\theta}{k_{2}}+\frac{\gamma N}{2 k_{2}}=\frac{1+\gamma N}{2}+\frac{3 \beta+2 \gamma N}{2 k_{2}}$. When the parameters $k_{1}, k_{2}$ and $\lambda$ are chosen according to (6), it follows from (17) that

$$
\begin{aligned}
\dot{V}(t) \leq & -\sigma \sum_{i=1}^{N}\left|p_{i}(t)\right|^{2}-\sigma \sum_{i=1}^{N}\left|v_{i}(t)-v_{i}^{*}(t)\right|^{2}-\sigma e^{2}(t)+\frac{\bar{l} N k_{1}^{2}}{2} \sum_{i=1}^{N}\left|\xi_{i}^{x}(t)\right|^{2} \\
& +\frac{1}{2 k_{2}^{2}} \sum_{i=1}^{N}\left(k_{1}^{2}\left|\xi_{i}^{v}(t)\right|^{2}+\left|\xi_{i}^{d}(t)\right|^{2}\right)+\frac{|| Q B_{d}||^{2}}{2} \sum_{i=1}^{N}\left|d_{i}^{(q-1)}(t)\right|^{2},
\end{aligned}
$$

where $\sigma$ and $\bar{l}$ are given in Theorem 1 .

Since $\xi_{i}^{T}(t) \Phi_{i} \xi_{i}(t) \leq \mu_{0} e^{-\mu_{1} t}+\mu_{2}$ for $t \geq 0$, we have that $\left\|\xi_{i}(t)\right\|^{2} \leq \frac{\mu_{0} e^{-\mu_{1} t}+\mu_{2}}{\lambda_{\min }\left(\Phi_{i}\right)}$. Letting $\xi=\operatorname{col}\left\{\xi_{1}, \cdots, \xi_{N}\right\}$, we get $\|\xi(t)\|^{2}=\left\|\xi^{x}(t)\right\|^{2}+\left\|\xi^{v}(t)\right\|^{2}+\left\|\xi^{d}(t)\right\|^{2} \leq \bar{\mu} e^{-\mu_{1} t}+\tilde{\mu}$, where $\bar{\mu}=\sum_{i=1}^{N} \frac{\mu_{0}}{\lambda_{\min }\left(\Phi_{i}\right)}$ and $\tilde{\mu}=\sum_{i=1}^{N} \frac{\mu_{2}}{\lambda_{\min }\left(\Phi_{i}\right)}$.

Noting that $\left|d_{i}^{(q-1)}(t)\right| \leq \bar{d}, \forall i \in \Gamma$ in Assumption 1, we obtain

$$
\dot{V}(t) \leq-\sigma \sum_{i=1}^{N}\left|p_{i}(t)\right|^{2}-\sigma \sum_{i=1}^{N}\left|v_{i}(t)-v_{i}^{*}(t)\right|^{2}-\sigma e^{2}(t)+\sigma_{1}\left(\bar{\mu} e^{-\mu_{1} t}+\tilde{\mu}\right)+\frac{\left.N \| Q B_{d}\right)\left.\right|^{2} \bar{d}^{2}}{2}
$$

where $\sigma_{1}$ is given in the statement of Theorem 1 .

Since $p_{i}=\sum_{j=1}^{N} l_{i j} x_{j}, i \in \Gamma$, it can be obtained that $\sum_{i=1}^{N} p_{i}^{2}=x^{T} L^{2} x$. Following Lemma 1 , we have $L \mathbf{1}_{N}=0$, which leads to $\mathbf{1}_{N}^{T} L \mathbf{1}_{N}=\left(L^{\frac{1}{2}} \mathbf{1}_{N}\right)^{T} L^{\frac{1}{2}} \mathbf{1}_{N}=0$. Thus, $L^{\frac{1}{2}} \mathbf{1}_{N}=0$, which means $\mathbf{1}_{N}^{T} L^{\frac{1}{2}} x=0$. According to Lemma 1 , if $\mathbf{1}_{N}^{T} L^{\frac{1}{2}} x=0$, then $\sum_{i=1}^{N} p_{i}^{2}=\left(L^{\frac{1}{2}} x\right)^{T} L\left(L^{\frac{1}{2}} x\right) \geq \lambda_{2} x^{T}(t) L x=2 \lambda_{2} V_{0}$. In addition, there holds $V_{e} \leq \frac{1}{2 \lambda_{M}(Q)} e^{2}$. Therefore, it can be shown that

$$
\begin{aligned}
V(t) & =V_{0}(t)+\sum_{i=1}^{N} V_{i}(t)+V_{e}(t) \\
& \leq \sigma_{2}\left(\sum_{i=1}^{N} p_{i}^{2}(t)+\sum_{i=1}^{N}\left|v_{i}(t)-v_{i}^{*}(t)\right|^{2}+e^{2}(t)\right),
\end{aligned}
$$

where $\sigma_{2}$ is defined in the statement of Theorem 1 .

Substituting (20) into (19), we get

$$
\dot{V}(t) \leq-\frac{\sigma}{\sigma_{2}} V(t)+\sigma_{1}\left(\bar{\mu} e^{-\mu_{1} t}+\tilde{\mu}\right)+\frac{\left.N \| Q B_{d}\right) \|^{2} \bar{d}^{2}}{2} .
$$

By the comparison lemma in [42], it follows from (21) that

$$
V(t) \leq e^{-\frac{\sigma}{\sigma_{2}} t} V(0)+\sigma_{1} \bar{\mu} \int_{0}^{t} e^{-\frac{\sigma}{\sigma_{2}}(t-s)} e^{-\mu_{1} s} \mathrm{~d} s+\int_{0}^{t} e^{-\frac{\sigma}{\sigma_{2}}(t-s)}\left(\sigma_{1} \tilde{\mu}+\frac{N\left\|Q B_{d}\right\|^{2} \vec{d}^{2}}{2}\right) \mathrm{d} s .
$$

When $\frac{\sigma}{\sigma_{2}}=\mu_{1}$, we get

$$
V(t) \leq e^{-\frac{\sigma}{\sigma_{2}} t} V(0)+\sigma_{1} \bar{\mu} t e^{-\frac{\sigma}{\sigma_{2}} t}+\frac{\sigma_{2}}{\sigma}\left(1-e^{-\frac{\sigma}{\sigma_{2}} t}\right)\left(\sigma_{1} \tilde{\mu}+\frac{\left.N \| Q B_{d}\right) \|^{2} \vec{d}^{2}}{2}\right) .
$$


Otherwise, we have

$$
V(t) \leq e^{-\frac{\sigma}{\sigma_{2}} t} V(0)+\frac{\sigma_{1} \sigma_{2} \bar{\mu}}{\sigma-\sigma_{2} \mu_{1}}\left(e^{-\mu_{1} t}-e^{-\frac{\sigma}{\sigma_{2}} t}\right)+\frac{\sigma_{2}}{\sigma}\left(1-e^{-\frac{\sigma}{\sigma_{2}} t}\right)\left(\sigma_{1} \tilde{\mu}+\frac{\left.N \| Q B_{d}\right) \|^{2} \bar{d}^{2}}{2}\right) .
$$

Finally, it can be obtained by (23) and (24) that $V(t)$ asymptotically converges to a bounded region centered at the origin with radius $\frac{\left.\sigma_{2}\left(2 \sigma_{1} \tilde{\mu}+N \| Q B_{d}\right) \|^{2} \vec{d}^{2}\right)}{2 \sigma}$ as time approaches infinity. According to the definition of $V$ and Assumption 2 , we get that the estimation errors and the output consensus errors asymptotically converge into the ball. Moreover, by carefully choosing the appropriate parameters $\lambda, k_{1}$ and $k_{2}$, we can adjust the radius to be arbitrarily small. As a conclusion, the output practical consensus problem is achieved under the proposed event-triggered consensus.

Remark 5 It should be first pointed out that the convergent radius in Theorem 1 will not go to zero as $\mu_{2}$ approaches zero according to the analysis. Recall that the radius is equal to $\frac{\left.\sigma_{2}\left(2 \sigma_{1} \tilde{\mu}+N \| Q B_{d}\right) \|^{2} \tilde{d}^{2}\right)}{2 \sigma}$, where $\tilde{\mu}=\sum_{i=1}^{N} \frac{\mu_{2}}{\lambda_{\min }\left(\Phi_{i}\right)}$. It can be deduced that the first part $2 \sigma_{1} \tilde{\mu}$ in the bracket will be zero when $\mu_{2}$ approaches zero, but the second part $\left.N \| Q B_{d}\right) \|^{2} \vec{d}^{2}$ in the bracket does not, because the undesirable influence of the time-varying disturbance can not be completely rejected in the steady state due to the sampling form of the proposed protocol.

\subsection{The Minimum Inter-Event Interval}

In this part, a detail analysis is given to guarantee the avoidance of the Zeno phenomenon.

Theorem 2 When the multi-agent systems (1) satisfy the Assumptions 1 and 2, under the proposed event-triggered output consensus protocol (2), (4) and (5), there exists a positive minimum inter-event interval between any two consecutive events for all agents, i.e., the Zeno phenomenon can be avoided. $\hat{d}_{i}(t)$.

Proof. By (5), we have $u_{i}(t)=-k_{1} k_{2} p_{i}(t)-k_{1} v_{i}(t)-k_{1} k_{2} \sum_{j=1}^{N} a_{i j}\left(\xi_{i}^{x}(t)-\xi_{j}^{x}(t)\right)-k_{1} \xi_{i}^{v}(t)-\xi_{i}^{d}(t)+k_{1} e_{i, 1}(t)-$ Considering (1) and (2), we get

$$
\begin{aligned}
\dot{x}_{i}(t)= & v_{i}(t), \\
\dot{\hat{v}}_{i}(t)= & \dot{z}_{i}^{1}(t)+\beta_{1} \dot{x}_{i}(t)=\beta_{1} e_{i, 1}(t)+\hat{d}_{i}(t)+u_{i}(t) \\
= & -k_{1} k_{2} p_{i}(t)-k_{1} v_{i}(t)-k_{1} k_{2} \sum_{j=1}^{N} a_{i j}\left(\xi_{i}^{x}(t)-\xi_{j}^{x}(t)\right) \\
& -k_{1} \xi_{i}^{v}(t)-\xi_{i}^{d}(t)+k_{1} e_{i, 1}(t)+\beta_{1} e_{i, 1}(t), \\
\dot{\hat{d}}_{i}(t)= & \beta_{2} e_{i, 1}(t)-e_{i, 3}(t)+\dot{d}_{i}(t) .
\end{aligned}
$$

For agent $i$, if the latest event happens at the instant $t_{i}^{k}$, then $x_{i}\left(t_{i}^{k}\right), \hat{v}_{i}\left(t_{i}^{k}\right)$ and $\hat{d}_{i}\left(t_{i}^{k}\right)$ keep the same in $\left[t_{i}^{k}, t_{i}^{k+1}\right)$, which leads to that $\dot{\xi}_{i}(t)=-\left[\dot{x}_{i}(t), \dot{\hat{v}}_{i}(t), \dot{\hat{d}}_{i}(t)\right]^{T}$.

In addition, $\left|v_{i}\right| \leq\left|v_{i}-v_{i}^{*}\right|+\left|v_{i}^{*}\right| \leq \sqrt{2} k_{2} \sqrt{V_{i}}+k_{2}\left|p_{i}\right|$. From Theorem 1, it follows that the estimation errors and the output consensus errors are uniformly ultimately bounded for $t \geq 0$ under the proposed consensus protocol. Recalling that $\left|d_{i}^{(j)}(t)\right| \leq \theta$ for $t \geq 0$ and $j=0, \cdots, q-1$ given in Assumption 1, we can obtain that there must exist a positive constant $M, \forall i \in \Gamma$, such that $\left\|\dot{\xi}_{i}(t)\right\| \leq M$ holds, $\forall t \in[0, \infty)$. Then,

$$
\left\|\xi_{i}(t)\right\| \leq \int_{t_{i}^{k}}^{t}\left\|\dot{\xi}_{i}(s)\right\| d s \leq M\left(t-t_{i}^{k}\right), \forall i \in \Gamma \text {. }
$$

With the help of (26) and the even-triggering condition (4), we have that the next event happens as soon as $\xi_{i}^{T}(t) \Phi_{i} \xi_{i}(t)$ crosses the threshold $\mu_{0} e^{-\mu_{1} t}+\mu_{2}$, which means that the next event is not triggered before $M\left(t-t_{i}^{k}\right)=$ $\frac{\mu_{0} e^{-\mu_{1} t}+\mu_{2}}{\lambda_{\min }\left(\Phi_{i}\right)}$. Hence, we can get a lower bound on the inter-event interval $\left[t_{i}^{k}, t_{i}^{k+1}\right)$, which is defined by the solution of the equation $M T=\frac{\mu_{0} e^{-\mu_{1}\left(t_{i}^{k}+T\right)}+\mu_{2}}{\lambda_{\min }\left(\Phi_{i}\right)}$. For all $t_{i}^{k} \geq 0$, it has that the solution $T\left(t_{i}^{k}\right) \geq T$, where $T$ is strictly positive and defined by $M T=\frac{\mu_{2}}{\lambda_{\min }\left(\Phi_{i}\right)}$. Finally, it can be concluded that there must exist a positive minimum inter-event interval for all agents, and the Zeno phenomenon can be successfully excluded. 


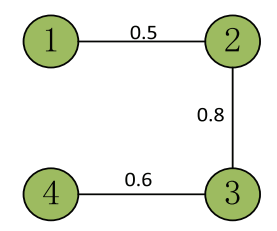

Figure 2: The communication topology for the second-order multi-agent systems.

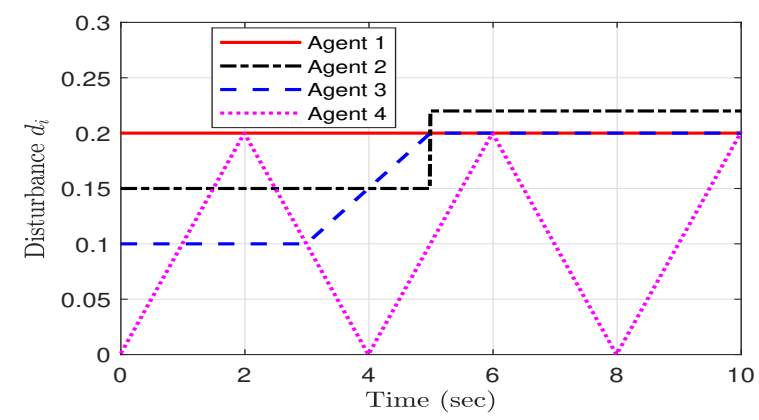

Figure 3: The curves of the time-varying disturbances.
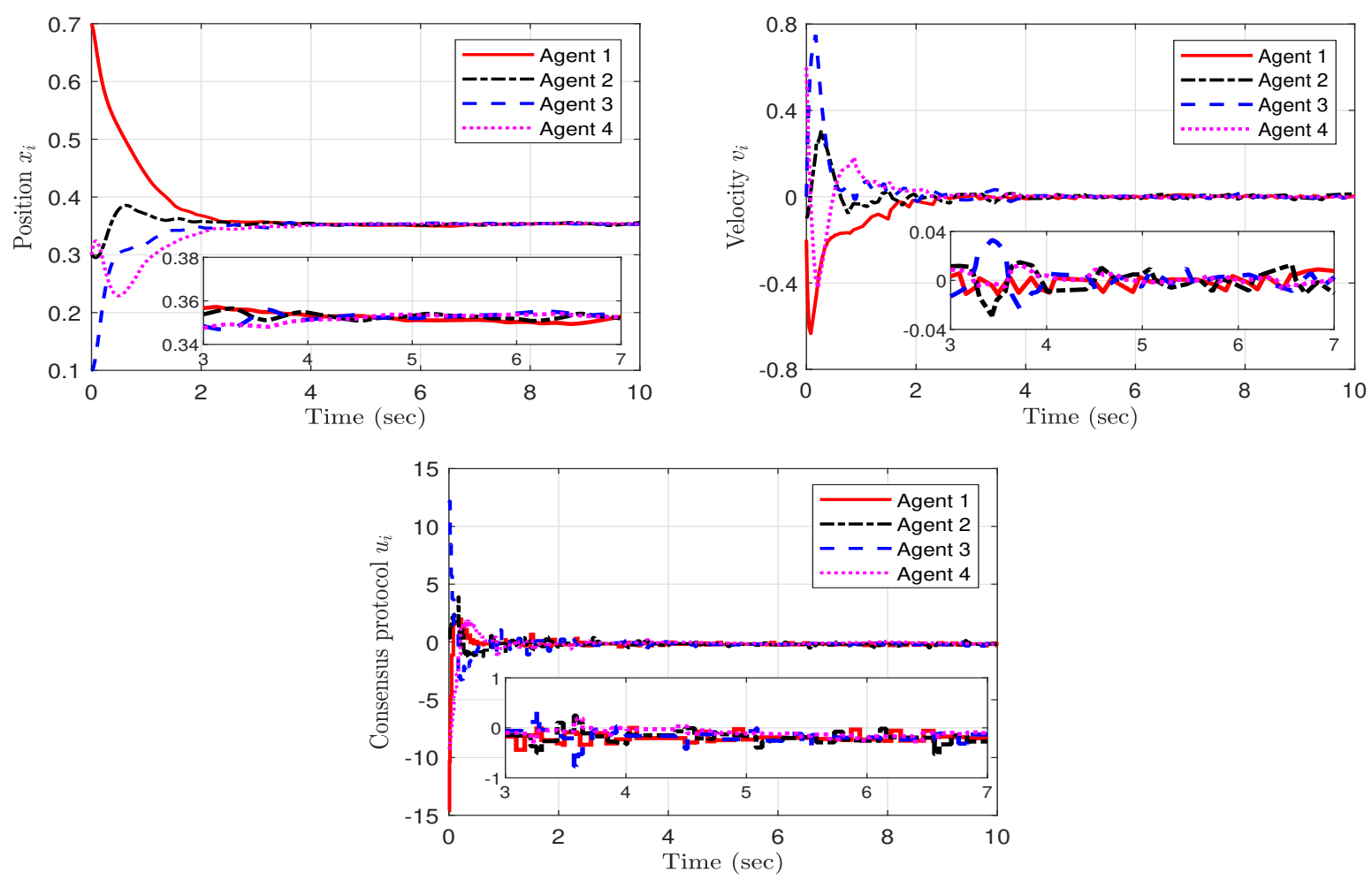

Figure 4: The trajectories of the states and the consensus protocol of each agent. 

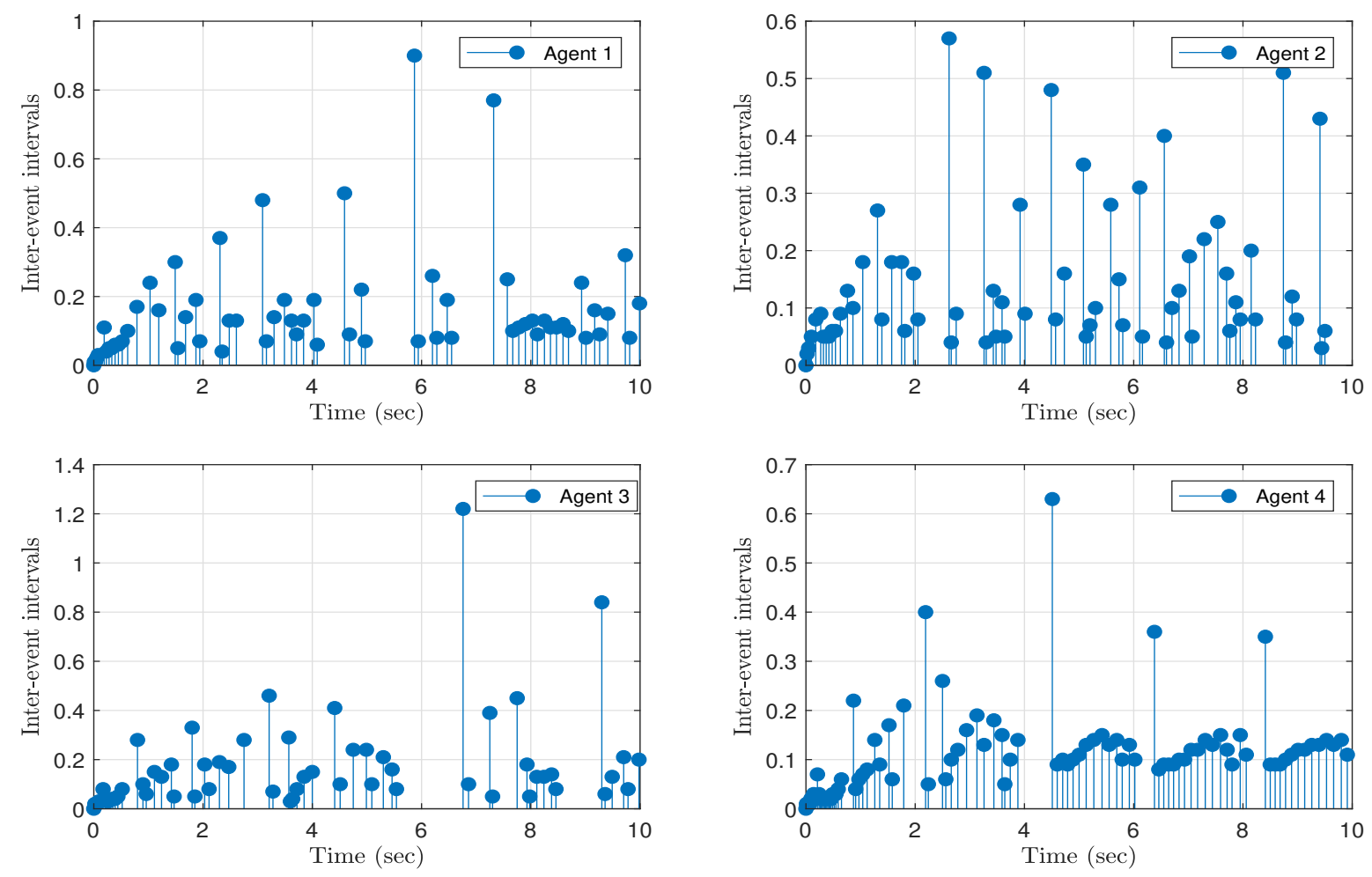

Figure 5: The event-triggering instants and the inter-event intervals for each agent under the proposed event-triggering mechanism.

\section{Simulations}

In order to demonstrate the effectiveness of on the proposed event-triggered consensus protocol, the simulation results are presented in this part. Fig. 2 depicts the communication topology graph $G$ with 4 agents, where the numbers along the edges stand for the weights of adjacency agent.

In the simulations, the parameters of the proposed output consensus strategy are set by $k_{1}=4, k_{2}=12.25$ and $\left[\beta_{1}, \beta_{2}, \beta_{3}\right]=[15,75,125]$. The parameters of the proposed event-triggering conditions are given by $\left[\mu_{0}, \mu_{1}, \mu_{2}\right]=$ $[0.1,-1.5,0.001]$, and the weight matrix parameters are chosen as $\Phi_{1}=\operatorname{diag}\{11,7,1\}, \Phi_{2}=\operatorname{diag}\{11,8,2\}, \Phi_{3}=$ $\operatorname{diag}\{9,5,3\}$ and $\Phi_{4}=\operatorname{diag}\{12,10,5\}$ for the different agents.

The time-varying disturbances $d_{i}(t), i=1, \cdots, 4$, are described in Fig. 3. The states and the consensus protocols are depicted in Fig. 4. It can be observed from Fig. 4 that the output of all the agents asymptotically achieve the practical consensus under the proposed output consensus strategy, since the disturbance estimation and compensation technique can reject the undesirable influence caused by the time-varying disturbances via the reduced-order GPIO. Fig. 5 displays the inter-event intervals and the event-triggering instants for each agent under the proposed event-triggering condition. Finally, compared with time-triggered consensus protocol, the proposed event-triggered consensus disturbance rejection strategy can significantly save the communication resource while maintaining a desirable consensus performance.

\section{Conclusions}

In order to solve the practical output consensus problem while saving the communication resource, a new eventtriggered consensus disturbance rejection protocol has been proposed for second-order multi-agent system via reducedorder GPIO in this study. By using the measurement and the control signal, the proposed reduced-order observer can accurately estimate the unknown states and the time-varying disturbances. Then, we developed a new event-triggered 
consensus disturbance rejection protocol to save the communication resource while maintaining a desirable consensus performance. The proposed event-triggering mechanism only requires the sampled information instead of the continuous information from its neighbors for each agent. In the presence of time-varying disturbances, both of the consensus performance and the communication resource utilization can be significantly improved by using the disturbance estimation and compensation technique. The delicate consensus analysis has demonstrated that by carefully choosing the appropriate parameters, the practical output consensus problem can be solved, and the avoidance of the Zeno behavior can be guaranteed in spite of time-varying disturbances. Finally, the effectiveness of the proposed consensus protocol was demonstrated by the simulation results. In the future, we will consider to extend the result of this paper to the cases of directed graph or jointly connected graph.

\section{Acknowledgement}

This work was supported in part by the National Natural Science Foundation of China under Grant 61973081, Grant 61973080, and Grant 61873060, in part by the Natural Science Foundation of Jiangsu Province under Grant BK20190061, in part by the Post-Doctoral Innovation Talent Support Program of China under Grant BX20200140.

\section{References}

[1] H. Zhang, G. Feng, H. Yan, Q. Chen, Observer-based output feedback event-triggered control for consensus of multi-agent system, IEEE Trans. Ind. Electron. 61 (9) (2014) 4885-4894.

[2] G.S. Seyboth, D.V. Dimarogonas, K.H. Johansson, Event-based broadcasting for multi-agent average consensus, Automatica 49 (1) (2013) 245-252.

[3] Z. Ding, Distributed adaptive consensus output regulation of network-connected heterogeneous unknown linear systems on directed graphs, IEEE Trans. Autom. Control 62 (9) (2017) 4683-4690.

[4] X.-M. Zhang, Q.-L. Han, X. Yu, Survey on recent advances in networked control systems, IEEE Trans. Ind. Informat. 12 (5) (2016) 17401752.

[5] C. Li, X. Yu, W. Yu, T. Huang, Z. Liu, Distributed event-triggered scheme for economic dispatch in smart grids, IEEE Trans. Ind. Informat. 12 (5) (2016) 1775-1785.

[6] Z.-W. Liu, G. Wen, X. Yu, Z.-H. Guan, T. Huang, Delayed impulsive control for consensus of multiagent systems with switching communication graphs, IEEE Trans. Cybern. 50 (7) (2020) 3045-3055.

[7] B. Zhang, Y. Jia, J. Du, J. Zhang, Finite-time synchronous control for multiple manipulators with sensor saturations and a constant reference, IEEE Trans. Control Syst. Technol. 22 (3) (2014) 1159-1165.

[8] K.J. Astrom, B. Wittenmark, Computer-Controlled Systems: Theory and Design, 3rd ed. Englewood Cliffs, NJ: Prentice-Hall, 1997.

[9] D.J. Stilwell, B.E. Bishop, Platoons of underwater vehicles, IEEE Control Syst. Mag. 20 (6) (2000) 45-52.

[10] Z. Gu, D. Yue, J. Liu, Z. Ding, Z. $H_{\infty}$ tracking control of nonlinear networked systems with a novel adaptive event-triggered communication scheme, J. Franklin Inst. 354 (8) (2017) 3540-3553.

[11] X.-C. Jia, X.-B. Chi, Q.-L. Han, N.-N. Zheng, Event-triggered fuzzy $H_{\infty}$ control for a class of nonlinear networked control systems using the deviation bounds of asynchronous normalized membership functions, Inf. Sci. 259 (2014) 100-117.

[12] J. Sun, J. Yang, S. Li, W. X. Zheng. Sampled-data-based event-triggered active disturbance rejection control for disturbed systems in networked environment. IEEE Trans. Cybern. 49 (2) (2019) 556-566.

[13] L. Xing, C. Wen, F. Guo, Z. Liu, H. Su, Event-based consensus for linear multiagent systems without continuous communication, IEEE Trans. Cybern. 47 (8) (2017) 2132-2142.

[14] J. Sun, J. Yang, S. Li, W. X. Zheng, Output-based dynamic event-triggered mechanisms for disturbance rejection control of networked nonlinear systems, IEEE Trans. Cybern. 50 (5) (2020) 1978-1988.

[15] J. Yang, J. Sun, W. X. Zheng, S. Li, Periodic event-triggered robust output feedback control for nonlinear uncertain systems with time-varying disturbance, Automatica 94 (2018) 324-333.

[16] X.-M. Zhang, Q.-L. Han, B.-L. Zhang, An overview and deep investigation on sampled-data-based event-triggered control and filtering for networked systems, IEEE Trans. Ind. Informat.13 (1) (2017) 4-16.

[17] L. Ding, Q.-L. Han, X. Ge, X.-M. Zhang, An overview of recent advances in event-triggered consensus of multiagent systems, IEEE Trans. Cybern. 48 (4) (2018) 1110-1123.

[18] H. Li, X. Liao, T. Huang, W. Zhu, Event-triggering sampling based leader-following consensus in second-order multi-agent systems, IEEE Trans. Autom. Control 60 (7) (2015) 1998-2003.

[19] X. Yin, D. Yue, S. Hu, Distributed event-triggered control of discrete-time heterogeneous multi-agent systems, J. Franklin Inst. 350 (3) (2013) 651-669.

[20] H. Zhang, D. Yue, X. Yin, S. Hu, C. Dou, Finite-time distributed event-triggered consensus control for multi-agent systems. Inf. Sci. 339 ( 2016) 132-142.

[21] S. Chen, J. Guan, Y. Gao, H. Yan, Observer-based event-triggered tracking consensus of non-ideal general linear multi-agent systems, J. Franklin Inst. 356 (17) (2019) 10355-10367.

[22] X. Liang, H. Wang, Y.-H. Liu, W. Chen, G. Hu, J. Zhao, Adaptive task-space cooperative tracking control of networked robotic manipulators without task-space velocity measurements, IEEE Trans. Cybern. 46 (10) (2016) 2386-2398. 
[23] D. Zhao, T. Dong, Reduced-order observer-based consensus for multi-agent systems with time delay and event trigger strategy, IEEE Access 5 (2017) 1263-1271.

[24] L. Zhou, J. She, S. Zhou, Robust $H_{\infty}$ control of an observer-based repetitive-control system, J. Franklin Inst. 355 (12) (2018) $4952-4969$.

[25] E.W. Zurita-Bustamante, J. Linares-Flores, E. Guzmán-Ramírez, H. Sira-Ramírez, A comparison between the gpi and pid controllers for the stabilization of a DC-DC 'buck' converter: A field programmable gate array implementation, IEEE Trans. Ind. Electron. 58 (11) (2011) 5251-5262.

[26] J. Sun, J. Yang, W. X. Zheng, S. Li, GPIO-based robust control of nonlinear uncertain systems under time-varying disturbance with application to DC-DC converter, IEEE Trans. Circuits Syst., II: Exp. Briefs 63 (11) (2016) 1074-1078.

[27] J. Sun, Z. Geng, Y. Lv, Z. Li, Z. Ding, Distributed adaptive consensus disturbance rejection for multi-agent systems on directed graphs, IEEE Trans. Control Netw. Syst. 5 (1) (2018) 629-639.

[28] W. Liu, J. Huang, Event-triggered cooperative global robust practical output regulation for second-order uncertain nonlinear multiagent systems, IEEE Trans. Neural Netw. Learn. Syst. 29 (11) (2018) 5486-5498.

[29] H. Cai, J. Huang, The leader-following consensus for multiple uncertain Euler-Lagrange systems with an adaptive distributed observer, IEEE Trans. Autom. Control 61 (10) (2018) 3152-3157.

[30] X. Wang, S. Li, X. Yu, J. Yang, Distributed active anti-disturbance consensus for leader-follower higher-order multi-agent systems with mismatched disturbances, IEEE Trans. Autom. Control 62 (11) (2017) 5795-5801.

[31] Z. Ding, Adaptive consensus output regulation of a class of nonlinear systems with unknown high-frequency gain, Automatica 51 (2015) 348-355.

[32] Y. Su, J. Huang, Cooperative output regulation of linear multi-agent systems, IEEE Trans. Autom. Control 57 (4) (2012) $1062-1066$.

[33] Z. Zhao, Y. Hong, Z. Lin, Semi-global output consensus of a group of linear systems in the presence of external disturbances and actuator saturation: An output regulation approach, Int. J. Robust Nonlin. Control 26 (7) (2016) 1353-1375.

[34] W. Hu, L. Liu, G. Feng, Output consensus of heterogeneous linear multi-agent systems by distributed event-triggered/self-triggered strategy, IEEE Trans. Cybern. 47 (8) (2017) 1914-1924.

[35] X. Wang, D. Xu, H. Ji, Robust almost output consensus in networks of nonlinear agents with external disturbances, Automatica 70 (2016) 303-311.

[36] D.N. Borgers, W.P.M.H. Heemels, Event-separation properties of event-triggered control systems, IEEE Trans. Autom. Control 59 (10) (2014) 2644-2656.

[37] C. Jiang, H. Du, W. Zhu, L. Yin, X. Jin, G. Wen, Synchronization of nonlinear networked agents under event-triggered control, Inf. Sci. 459 (2018) 317-326.

[38] X. Shao, H. Wang, Back-stepping active disturbance rejection control design for integrated missile guidance and control system via reducedorder ESO, ISA Trans. 57 (2015) 10-22.

[39] R. Olfati-Saber, R.M. Murray, Consensus problems in networks of agents with switching topology and time-delays, IEEE Trans. Autom. Control 49 (9) (2004) 1520-1533.

[40] R.A. Horn, C.R. Johnson, Matrix Analysis. Cambridge, U.K.: Cambridge Univ. Press, 1985.

[41] W. Rudin, Real and Complex Analysis, 3rd ed. New York: McGraw Hill Higher Education, 1986.

[42] H.K. Khalil. Nonlinear Systems, 3rd ed. Upper Saddle River, NJ: Prentice-Hall, 2002. 